www.jmscr.igmpublication.org

Impact Factor 5.244

Index Copernicus Value: 5.88

ISSN (e)-2347-176x ISSN (p) 2455-0450

crossref DOI: _http://dx.doi.org/10.18535/jmscr/v4i4.46

\title{
A Rare Case of Well Differentiated Papillary Mesothelioma of the Tunica Vaginalis
}

\author{
Megha Yadav', Mukesh Agrawal ${ }^{2}$, Jaya Bhaskar Reddy ${ }^{3}$,Sujani Madabhushi ${ }^{4}$, \\ Mujeeb Siddiqui ${ }^{5}$ \\ ${ }^{1,3,4,5}$ Department of Pathology, VIMTA LABS Ltd, Cherlapally, Hyderabad, Telangana, India. \\ ${ }^{2}$ Lab Director, VIMTA LABS Ltd, Cherlapally, Hyderabad, Telangana, India \\ Corresponding Author \\ Megha Yadav
}

Head, Dept of Pathology, VIMTA Labs Ltd, Phase-II Cherlapally, Hyderabad- 500051, Telangana, India Emaildrmegha1852@gmail.com

\begin{abstract}
Well Differentiated Papillary Mesothelioma (WDPM) is an uncommon neoplasm of the paratesticular area usually presents as hydrocele. Here we report a rare case of benign papillary mesothelioma of the tunica vaginalis presented as hydrocele with singly polypoidal grossly papillary lesion with multiple foci in the hydrocele sac. Histologically tumor shows branching papillary structures with fibro vascular core and cytologically bland nuclei without any mitosis, necrosis or area of invasion. In addition histochemistry is done to differentiate it with other papillary neoplasm. WDPM must be distinguished from the aggressive lesion to avoid unnecessary treatment.

Keywords- Well Differentiated Papillary Mesothelioma (WDPM), Paratesticular area, Hydrocele, Tunica Vaginalis.
\end{abstract}

\section{Introduction}

Well Differentiated Papillary Mesothelioma (WDPM) is an unusual variant of epithelial mesothelioma.

It occurs mainly in the peritoneum, and this tumor is most commonly seen in young women who have no history of asbestos exposure ${ }^{[1]}$. WDPM occurs rarely at other sites including pericardium, pleura and tunica vaginalis. Unlike the conclusive link between asbestos exposure and diffuse malignant mesothelioma an association of asbestos and WDPM has not been definitely established ${ }^{[2]}$.
The majority of tumors with histological features of WDPM have behaved in a benign or indolent fashion. However several WDPM have pursued a more aggressive course resulting in death after the development of DMM ${ }^{[2]}$. We report a case of WDPM in a 50 year old male presented with hydrocele.

\section{Case Report}

A 50 year old patient presented with scrotal swelling in a surgical OPD. The swelling was gradually increasing in the size within a period of 8 months. No history of trauma or asbestos 


\section{JMSCR Vol||04||Issue||04||Page 10252-10255||April}

exposure. Local examination revealed hydrocele. Ultrasonography of the testicular region showed papillary growth of $1.5 \mathrm{~cm}$ in the paratesticular area. Orchidectomy was done and sent for histopathological diagnosis.

Macroscopically testis was normal with one pedunculated papillary mass of $1.5 \times 1 \mathrm{~cm}$ in diameter found in the tunica vaginalis (Fig 1). The hydrocele sac was of $10 \times 6 \times 4 \mathrm{~cm}$. Cut section of the sac was showing diffuse infiltrating nodules of varying sizes ranging from 0.5 to $0.8 \mathrm{~cm}$. Hydrocele sac was showing hemorrhagic areas.

Microscopically the papillary mass and the nodules from the sac wall were showing multiple branching papillae with fibrovascular cores. These papillae were lined by single layer of flat cuboidal to columnar epithelium (Fig 2). No cellular pleomorphism, mitotic activity, necrosis or stromal invasion was noted. Immunohistochemistry of the tumor was showing positivity for EMA, Cytokeratin, Vimentin and calretinin.----of cells show positive for $\mathrm{p} 53$ and negative for CEA (Fig 3,4)

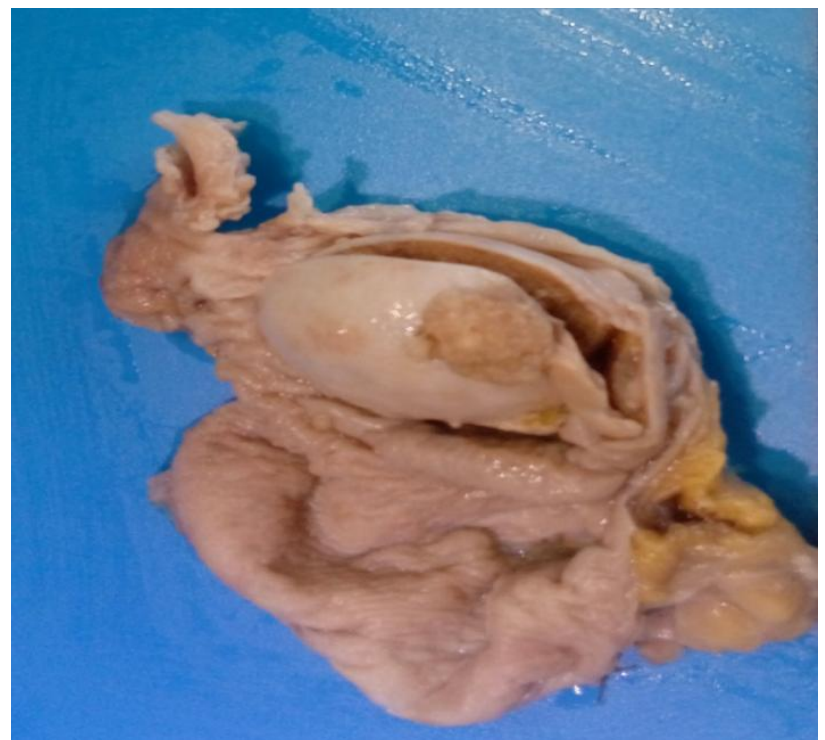

Fig 1. Pedunculated papillary mass of $1.5 \times 1 \mathrm{CM}$ in the tunica vaginalis

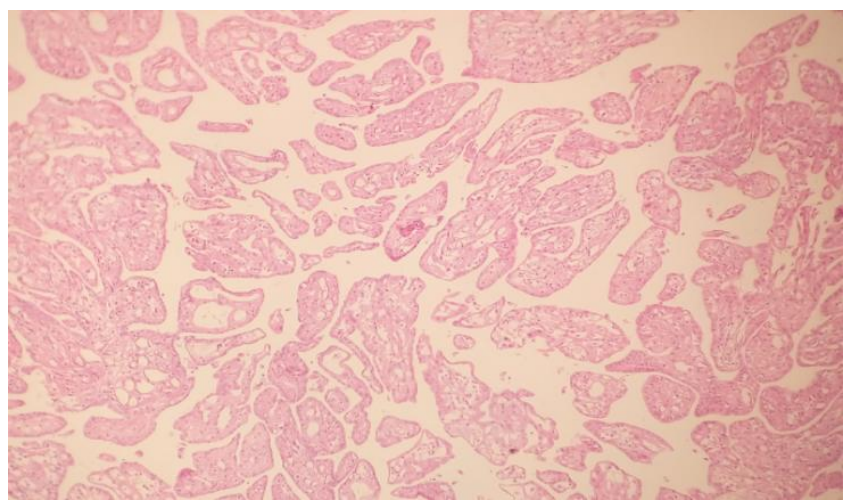

Fig 2. Papillary mass with branching papillae with fibrovascular cores lined by single layer of cuboidal epithelium (10x)

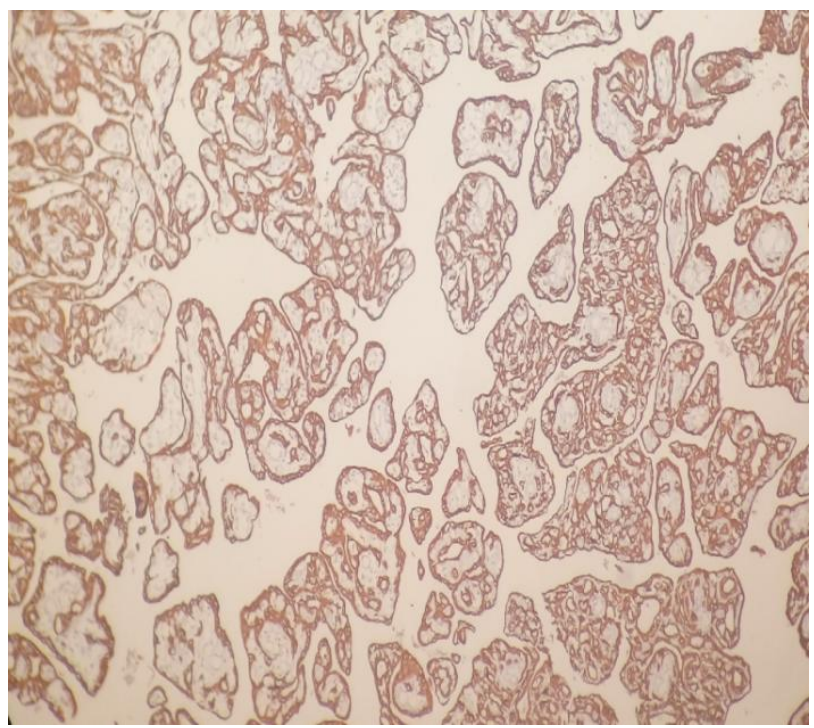

Fig 3. Positive for cytokeratin

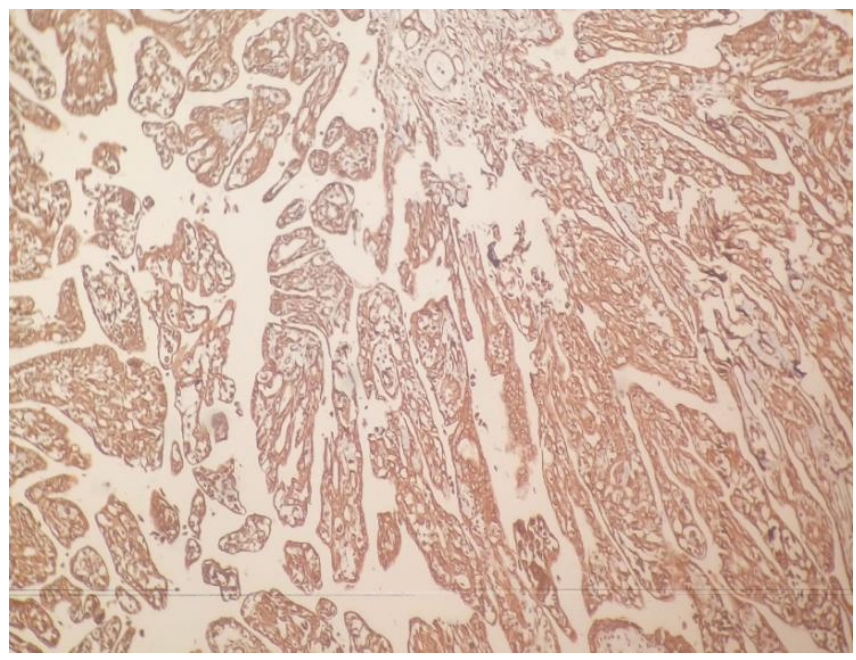

Fig 4. Positive for Vimentin

\section{Discussion}

Mesothelioma can arise from the pleura, peritoneum or paratesticular area in decreasing 
order of frequency. Paratesticular mesothelioma can arise from the tunica vaginalis formed by an out pouching of the abdominal peritoneum. Only $0.3-5 \%$ of cases of mesothelioma occur in the tunica vaginalis ${ }^{[3]}$.WDPM of tunica vaginalis is considered benign mesothelioma in the World Health Organization fascicle on the tumors of the urinary system and male genital organs, primarily due to indolent behavior. Main mesothelial lesion of the paratesticular area include mesothelial hyperplasia, mesothelial cyst, adenomatoid tumor, WDPM and malignant mesothelioma. Barbera and Rubino reported the first case of WDPM in 1957. To the best of our knowledge 19 cases of paratesticular WDPM have been published to date. WDPM were described in men between the ages of 18-70 years (median 56). All reported cases with WDPM had a good outcome with no evidence of disease recurrence or progression ${ }^{[4]}$. The definition of WDPM has been controversial, but most experts currently restrict the term only to localized, solitary and exophytic tumors of delicate papillae lined by bland cuboidal cells ${ }^{[2],[4],[5]}$.Some of the cases were designated as "papillary mesotheliomas with borderline features or localized mesotheliomas of low grade malignancy, cautioning about their low malignant potential, despite the benign follow -up ${ }^{[2],[4]}$.

Main differential diagnosis of WDPM includes mesothelial hyperplasia, malignant mesothelioma and papillary cystadenoma. Mesothelial hyperplasia sometimes also composed of papillary structure but often not contain fibrovascular core and they are always associated with the inflammation and reactive change [3],[6],[7]. Presence of invasion, marked cytological atypia, atypical mitosis, necrosis, big mass are the main differentiating features of WDPM and MM.

At immunohistochemical level, no study to date has compared the expression of different markers between diffuse malignant mesothelioma and well differentiated papillary mesothelioma. Rather studies have focused on differentiating diffuse malignant mesothelioma from reactive mesothelial proliferation. Although no single antibody has been shown to carry a discriminatory value, several markers have been reported to be more commonly expressed in diffuse malignant mesothelioma than in reactive mesothelium ${ }^{[8]}$.Calretinine, Pan ck, EMA and Vimentin shows positivity for the WDPM. CEA will be negative which may be the main differentiating feature to a papillary serous cystadenoma.

The etiology of WDPM has not been clearly elucidated. It has been suggested that proliferative lesions might be related to local trauma, herniorraphy or a long term hydrocele. A relationship between asbestos exposure and mesothelioma of the tunica vaginalis is postulated [3].

Our case had no asbestos exposure like the rest of the reported cases.

We describe a mesothelial neoplasm of the tunica vaginalis called as well differentiated papillary mesothelioma of tunica vaginalis. No complex architecture with stromal invasion or coagulative necrosis or increase in the mitotic activity is seen in our case. Sometimes WDPM may be confused with carcinomatosis on surgery, but can be readily distinguished microscopically. Although our case had no invasion, atypia, infiltrative growth or mitotic activity, we recommended close patient follow up because of the association of the multiple nodules in the hydrocele sac showing the same morphological features. The biological behavior of WDPM is not well known because of the limited number of cases.

\section{References}

1. B.Anil kumar, Sanjana Gogineni, Channareddy Suneetha, Kalyan Chakravarthy, B.Nissy Jacintha, "A rare case of papillary mesothelioma of peritoneal origin,'JOSR Journal of Dental and Medical Sciences., vol.6,Issue 5, pp.01-04.2013

2. Kelly J. Butnor, Thomas A. Sporn, Samuel P. Hammar, Victor L Roggli , "Well differentiated papillary mesothelioma,". 
Am J Surg Pathol., 25(10), pp. 13041309.2001

3. Seyda Erdogan, Arbil Acikalin, Handen Zaren, Gulfiliz Gonlusen, Susan Zorludemir, Volkan Izol, "Well Differentiated Papillary Mesothelioma of the Tunica Vaginalis: A Case Study and Review of the Literature," Korean J Pathol.,48, pp.225-228.2014

4. Kiril Trpkov, Richard Barr, Andrew Kulaga, Asli Yilmaz, "Mesothelioma of tunica vaginalis of uncertain malignant potential- an evolving concept: Case report and review of the literature,". Diagnostic pathology., 6:78.2011

5. Davis Dj, Woodward PJ : Pathology and Genetics of the Urinary system and Male Genital Organs. In World classification of Tumours .Edited by Eble J Sauter G, Epstein J, Sesterhenn I. Lyon: IARC Press; 2004:269

6. Chen JL, Hsu YH, “ Malignant mesothelioma of the tunica vaginalis testis: a case report and literature review," Kaohsiung J Med Sci., 25,pp.77-81.2002

7. Goel A, Agrawal A, Gupta R, Hari S, Dey $\mathrm{AB}$, " Malignant mesothelioma of the tunica vaginalis of the testis without exposure to asbestos," Cases J,1:310.2008

8. Fadi Brimo,Peter B Illei, Jonathan I Epstein, " Mesothelioma of the tunica vaginalis: a series of eight cases with uncertain malignant potential," Modern pathology., 23, pp. 1165-1172.2010 